\title{
Comparison of the NIST and BIPM Standards for Air Kerma in Medium-Energy X-Rays
}

\section{T. Burns}

Bureau International des Poids et Mesures, F-92312 Sèvres Cedex, France

and

\section{O'Brien}

National Institute of Standards and Technology,

Gaithersburg, MD 20899 USA

michelle.obrien@nist.gov
A comparison has been made of the airkerma standards for medium-energy x-rays of the National Institute of Standards and Technology (NIST) and the Bureau International des Poids et Mesures (BIPM). The comparison involved a series of measurements at the BIPM and the NIST using the air-kerma standards and three NIST reference-class transfer ionization chamber standards. Reference beam qualities in the range from $100 \mathrm{kV}$ to 250 $\mathrm{kV}$ were used. The results show the standards to be in reasonable agreement within the combined standard uncertainty of the comparison of $0.37 \%$, although a significant trend with radiation quality is observed and the possible sources discussed.
Key words: air kerma; free-air ionization chamber; primary standard; reference radiation qualities; medium-energy $\mathrm{x}$-rays; $\mathrm{x}$ ray calibration.

Accepted: August 18, 2006

Available online: http://www.nist.gov/jres

\section{Introduction}

An indirect comparison was made between the airkerma primary standards for medium-energy $\mathrm{x}$-rays of the National Institute of Standards and Technology (NIST) and the Bureau International des Poids et Mesures (BIPM). The measurements were conducted at both laboratories between November 2002 and June 2003 using the tungsten reference radiation qualities in the range from $100 \mathrm{kV}$ to $250 \mathrm{kV}$ recommended by the Consultative Committee for Ionizing Radiation (CCEMRI) [1]. Three NIST reference-class ionization chambers were used as transfer instruments for the comparison.

\section{Determination of the Air-Kerma Rate}

For a free-air ionization chamber standard with measuring volume $V$, the air-kerma rate is determined by the relation

$$
\dot{K}=\frac{I}{\rho_{\text {air }} V} \frac{W_{\text {air }}}{e} \frac{1}{1-g_{\text {air }}} \prod_{i} k_{i},
$$

where

$I / \rho_{\text {air }} V$ is the mass ionization current measured by the standard,

$W_{\text {air }}$ is the mean energy expended by an electron of charge $e$ to produce an ion pair in dry air,

$g_{\text {air }}$ is the fraction of the initial electron energy lost by bremsstrahlung production in air, and 
$\Pi k_{\mathrm{i}}$ is the product of the correction factors to be applied to the standard.

The values for the physical constants used in the determination of the air-kerma rate are given in Table 1.

Table 1. Physical constants used in the determination of the airkerma rate

\begin{tabular}{lcc}
\hline \hline $\begin{array}{l}\text { Physical } \\
\text { constant }\end{array}$ & Value & $\begin{array}{c}\text { Relative standard } \\
\text { uncertainty } \\
(\%)\end{array}$ \\
\hline$\rho_{\text {air }}{ }^{\mathrm{a}}$ & $1.293 \mathrm{~kg} \mathrm{~m}^{-3}$ & 0.01 \\
$W_{\text {air }} / e$ & $33.97 \mathrm{~J} \mathrm{C}^{-1}$ & 0.15 \\
\hline
\end{tabular}

${ }^{\mathrm{a}}$ Density of dry air at $273.15 \mathrm{~K}$ and $101325 \mathrm{~Pa}$.

\section{Characteristics of Chambers}

\subsection{Description of Air-Kerma Standards}

Both air-kerma standard chambers used in the comparison are parallel-plate free-air ionization chambers. The measuring volume $V$ is defined by the diameter of the chamber aperture and the length of the collecting plate. The BIPM air-kerma standard is described in Refs. [2] and [3]. The NIST Wyckoff-Attix chamber is described in Refs. [4] and [5]. The main dimensions, the measuring volume and the polarizing voltage for each chamber are given in Table 2.

Table 2. Main characteristics of the primary standards used in the comparison

\begin{tabular}{lll}
\hline \hline Characteristic & NIST & BIPM \\
\hline Air-path length/cm & 30.8 & 28.15 \\
Plate separation/cm & 20.0 & 18.0 \\
Collecting plate length/cm & 10.08 & 6.0004 \\
Aperture diameter/cm & 0.9999 & 0.9939 \\
Measuring volume $/ \mathrm{cm}^{3}$ & 7.915 & 4.6554 \\
Polarizing voltage $/ \mathrm{V}$ & -5000 & 4000 \\
\hline
\end{tabular}

\subsection{Description of Transfer Ionization Chambers}

Three NIST transfer chambers (NIST-T1, NIST-T2, NIST-T3, serial numbers 2022, 2023, and 260 respectively) were used for the comparison. Each was calibrated at the NIST before and after a series of calibrations at the BIPM in February 2003. All three transfer standards are spherical and constructed with a wall of air-equivalent plastic. Each has a nominal volume of
$3.6 \mathrm{~cm}^{3}$, an external diameter of $1.9 \mathrm{~cm}$ and a wall thickness of $0.25 \mathrm{~mm}$. A collecting voltage of $300 \mathrm{~V}$ was applied to each standard. Measurements were made using both polarities at each laboratory, although the comparison results are derived from the negative polarity measurements only.

\section{Comparison Details}

\subsection{Irradiation Facilities and Reference Beam Qualities}

The BIPM measurements were made at the BIPM medium-energy x-ray laboratory which, at the time of the comparison, housed a constant-potential generator (maximum usable generating potential $250 \mathrm{kV}$ ) and a tungsten-anode $\mathrm{x}$-ray tube with an inherent filtration of around $2.3 \mathrm{~mm}$ of aluminum. Both the generating potential and the tube current were stabilized using feedback systems constructed at the BIPM, resulting in high stability and eliminating the need for a transmission current monitor. For a well-behaved transfer instrument, the variation in the measured ionization current over the duration of a comparison was typically less than $0.04 \%$. The BIPM radiation qualities in the energy between $100 \mathrm{kV}$ and $250 \mathrm{kV}$ are those recommended by CCEMRI [1] and are given in Table 3 .

The NIST measurements were made using the Wyckoff-Attix free-air chamber in the NIST $300 \mathrm{kV}$ calibration facility. The $\mathrm{x}$-ray source at the time of the comparison was a $320 \mathrm{kV}$ x-ray generator with a metalceramic $\mathrm{x}$-ray tube, both supplied by Seifert-Pantak ${ }^{1}$. The $\mathrm{x}$-ray generator is a high-frequency, highly stabilized voltage source. The tungsten anode $\mathrm{x}$-ray tube, model MXR-321, has a beryllium window of thickness $3 \mathrm{~mm}$ and a focal spot $8.0 \mathrm{~mm}$ in diameter. The materials used for the filtration and for the measurement of HVL were at least $99.99 \%$ pure with thicknesses known with an uncertainty of $0.01 \mathrm{~mm}$. The output stability was monitored using a transmission ionization chamber. The reference radiation qualities used at the NIST were generated between $100 \mathrm{kV}$ to $250 \mathrm{kV}$ and are listed in Table 3.

\footnotetext{
${ }^{1}$ Certain commercial equipment, instruments, or materials are identified in this paper to foster understanding. Such identification does not imply recommendation or endorsement, nor does it imply that the equipment identified are necessarily the best available for the purpose.
} 
Volume 111, Number 5, September-October 2006

Journal of Research of the National Institute of Standards and Technology

Table 3. Characteristics of the reference radiation qualities used for the comparison

\begin{tabular}{|c|c|c|c|c|c|c|c|}
\hline \multirow[t]{2}{*}{$\begin{array}{l}\text { Reference } \\
\text { radiation }\end{array}$} & \multirow{2}{*}{$\begin{array}{c}\text { Generating } \\
\text { potential } \\
\mathrm{kV}\end{array}$} & \multicolumn{2}{|c|}{ Additional filtration } & \multicolumn{2}{|c|}{$\begin{array}{l}\text { Half-value } \\
\text { layer (HVL) }\end{array}$} & \multirow{2}{*}{$\begin{array}{c}\text { Attenuation } \\
\text { coeff } \mu_{\text {air }}^{\text {a }} \\
\mathrm{m}^{-1}\end{array}$} & \multirow{2}{*}{$\begin{array}{l}\text { Air-kerma } \\
\quad \text { rate } \\
\mathrm{mGy} \mathrm{s}^{-1}\end{array}$} \\
\hline & & $\mathrm{mm} \mathrm{Al}$ & $\mathrm{mm} \mathrm{Cu}$ & $\mathrm{mm} \mathrm{Al}$ & $\mathrm{mm} \mathrm{Cu}$ & & \\
\hline \multicolumn{8}{|l|}{ BIPM } \\
\hline $100 \mathrm{kV}$ & 100 & 1.2032 & & 4.027 & 0.148 & 0.0355 & 0.21 \\
\hline $135 \mathrm{kV}$ & 135 & & 0.2321 & & 0.494 & 0.0235 & 0.21 \\
\hline $180 \mathrm{kV}$ & 180 & & 0.4847 & & 0.990 & 0.0198 & 0.30 \\
\hline $250 \mathrm{kV}$ & 250 & & 1.5701 & & 2.500 & 0.0172 & 0.39 \\
\hline \multicolumn{8}{|l|}{ NIST } \\
\hline $100 \mathrm{kV}$ & 100 & 3.248 & & 3.943 & 0.149 & 0.0493 & 1.04 \\
\hline $135 \mathrm{kV}$ & 135 & 1.060 & 0.265 & & 0.496 & 0.0270 & 1.00 \\
\hline $180 \mathrm{kV}$ & 180 & 3.842 & 0.482 & & 1.003 & 0.0257 & 1.30 \\
\hline $250 \mathrm{kV}$ & 250 & 3.842 & 1.618 & & 2.502 & 0.0179 & 1.58 \\
\hline
\end{tabular}

${ }^{\mathrm{a}}$ Air attenuation coefficient at $293.15 \mathrm{~K}$ and $101325 \mathrm{~Pa}$, measured at the BIPM for an air path length of $270 \mathrm{~mm}$ and at the NIST for 308 mm.

\subsection{Correction Factors}

Although free-air chambers are designed to minimize or eliminate corrections to the measured ionization current, certain corrections are unavoidable. The correction factors applied to each free-air chamber and the associated uncertainties are listed in Tables 4 and 5 .

A correction must be made for the attenuation of the $\mathrm{x}$-ray fluence along the air path between the reference plane and the center of the collecting volume. The correction factor $k_{\mathrm{a}}$ is calculated using the measured airattenuation coefficients $\mu_{\text {air }}$, according to

$$
k_{\mathrm{a}}=\exp \left(\mu_{\text {air }} L\right)
$$

The effective attenuation path $\mathrm{L}$ varies with the temperature and pressure of the air in the chamber and so the values for $k_{\mathrm{a}}$ are corrected for this effect. All ionization measurements are also corrected for the temperature and pressure of the ambient air between the radiation source and the reference plane.

All measured ionization currents using the free-air chamber standards are corrected for ion recombination, $k_{\mathrm{s}}$. The ionization currents measured with the transfer standards are not corrected for ion recombination. However, since the air-kerma rates used at both facilities are low, minimal volume recombination occurs in the transfer chambers. This was demonstrated by making separate recombination measurement at the BIPM for two of the three transfer chambers. From these one can conclude that the different air-kerma rates at the NIST and the BIPM do not affect the comparison results by more than $0.02 \%$. The standard chambers are corrected for the humidity effect $k_{h}$ which is taken as 0.998 for both standards and all radiation qualities.

\subsection{Chamber Positioning and Measurement Procedure}

Each calibration was made by alternating between the transfer chamber and the standard free-air chamber, this being achieved by movement of the x-ray tube at the BIPM and by translation of the chambers at the NIST. At both laboratories alignment on the beam axis was measured to around $0.1 \mathrm{~mm}$ and this position was reproducible to better than $0.01 \mathrm{~mm}$, as observed by an alignment telescope. No correction is applied for the radial non-uniformity of each beam. The reference plane for each chamber was positioned at $1000 \mathrm{~mm}$ from the radiation source at the NIST and $1200 \mathrm{~mm}$ at the BIPM. The beam diameter in the reference plane was $60 \mathrm{~mm}$ at the NIST and $83 \mathrm{~mm}$ at the BIPM.

The leakage current was measured before and after each series of ionization current measurements and a correction made based on the mean of these leakage measurements. For all measurements the leakage current was less than $0.01 \%$ of the ionization current. For all chambers at the NIST a total of not less than 30 measurements with an integration time of $60 \mathrm{~s}$ were made. The statistical standard uncertainty of the current measurements was typically less than $0.1 \%$ for the NIST transfer chambers. At the BIPM each calibration coefficient was obtained from a total of 14 measurements of around $60 \mathrm{~s}$ performed with the BIPM standard and each transfer chamber. The statistical standard uncertainty of each calibration coefficient is less than 
Volume 111, Number 5, September-October 2006

Journal of Research of the National Institute of Standards and Technology

Table 4. Correction factors used in the comparison for the NIST Wyckoff-Attix standard

\begin{tabular}{lcccccc}
\hline \hline Correction factor & \multicolumn{3}{c}{ Generating potential (kV) } & \multicolumn{3}{c}{$\begin{array}{c}\text { Relative standard } \\
\text { uncertainty (\%) }\end{array}$} \\
& 100 & 135 & 180 & 250 & Type A & Type B \\
\hline Air attenuation $k_{\mathrm{a}}^{\mathrm{a}}$ & 1.0152 & 1.0083 & 1.0079 & 1.0055 & 0.12 & 0.02 \\
Scattered radiation $k_{\mathrm{sc}}$ & 0.9942 & 0.9952 & 0.9958 & 0.9969 & - & 0.07 \\
Electron loss $k_{\mathrm{e}}$ & 1.0000 & 1.0006 & 1.0027 & 1.0055 & - & 0.05 \\
Ion recombination $k_{\mathrm{s}}$ & 1.0004 & 1.0001 & 1.0006 & 1.0002 & 0.1 & - \\
Fluorescence $k_{\mathrm{fl}}$ & 0.9981 & 0.9991 & 0.9995 & 0.9999 & - & 0.03 \\
Aperture transmission $k_{1}^{\mathrm{b}}$ & 1.0000 & 1.0000 & 1.0000 & 1.0000 & - & 0.04 \\
Field distortion $k_{\mathrm{d}}$ & 1.0015 & 1.0015 & 1.0015 & 1.0015 & - & 0.2 \\
Polarity effect $k_{\mathrm{pol}}$ & 1.001 & 1.000 & 1.001 & 1.001 & 0.05 & - \\
Wall transmission $k_{\mathrm{p}}{ }^{\mathrm{b}}$ & 1.0000 & 1.0000 & 1.0000 & 1.0000 & - & 0.01 \\
Bremsstrahlung $1-g_{\text {air }}$ & 1.0000 & 1.0000 & 1.0000 & 1.0000 & - & 0.01 \\
Humidity $k_{h}$ & 0.998 & 0.998 & 0.998 & 0.998 & - & 0.03 \\
\hline
\end{tabular}

${ }^{\text {a }}$ These are nominal values for $T=293.15 \mathrm{~K}$ and $p=101325 \mathrm{~Pa}$. Each measurement is corrected using the air temperature and pressure measured at the time.

${ }^{\mathrm{b}}$ The uncertainties in the aperture and wall transmission corrections are negligible.

Table 5. Correction factors used in the comparison for the NIST Wyckoff-Attix standard

\begin{tabular}{lcccccc}
\hline \hline Correction factor & \multicolumn{3}{c}{ Generating potential (kV) } & \multicolumn{2}{c}{$\begin{array}{c}\text { Relative standard } \\
\text { uncertainty (\%) }\end{array}$} \\
& 100 & 135 & 180 & 250 & Type A & Type B \\
\hline Air attenuation $k_{\mathrm{a}}^{\mathrm{a}}$ & 1.0102 & 1.0067 & 1.0057 & 1.0049 & 0.03 & 0.01 \\
Scattered radiation $k_{\mathrm{sc}}^{\mathrm{b}}$ & 0.9952 & 0.9959 & 0.9964 & 0.9974 & - & 0.03 \\
Fluorescence $k_{\mathrm{fl}}^{\mathrm{b}}$ & 0.9985 & 0.9992 & 0.9994 & 0.9999 & - & 0.03 \\
Electron loss $k_{\mathrm{e}}^{\mathrm{b}}$ & 1.0000 & 1.0016 & 1.0043 & 1.0073 & - & 0.09 \\
Ion recombination $k_{\mathrm{s}}$ & 1.0004 & 1.0005 & 1.0005 & 1.0003 & 0.02 & 0.01 \\
Field distortion $k_{\mathrm{d}}$ & 1.0000 & 1.0000 & 1.0000 & 1.0000 & - & 0.07 \\
Aperture transmission $k_{1}$ & 0.9999 & 0.9998 & 0.9997 & 0.9996 & - & 0.01 \\
Wall transmission $k_{\mathrm{p}}$ & 1.0000 & 0.9999 & 0.9999 & 0.9988 & 0.01 & - \\
Bremsstrahlung $1-g_{\text {air }}$ & 0.9999 & 0.9999 & 0.9998 & 0.9997 & - & 0.01 \\
Humidity $k_{h}$ & 0.998 & 0.998 & 0.998 & 0.998 & - & 0.03 \\
\hline
\end{tabular}

${ }^{a}$ These are nominal values for $T=293.15 \mathrm{~K}$ and $p=101325 \mathrm{~Pa}$. Each measurement is corrected using the air temperature and pressure measured at the time.

${ }^{\mathrm{b}}$ Values for $k_{\mathrm{sc}}, k_{\mathrm{fl}}$, and $k_{\mathrm{e}}$ adopted in October 2003, based primarily on Monte Carlo calculations.

$0.02 \%$. Repeatability of the calibration coefficient on different days, after having removed and replaced the chamber, was typically $0.03 \%$.

The polarity correction for the transfer chambers was measured at the BIPM to be only typically 1.0006 for all transfer chambers. Only the negative polarity measurements are used for the comparison results.

All transfer ionization chamber current measurements were normalized to $293.15 \mathrm{~K}$ and $101325 \mathrm{~Pa}$. The transfer chamber currents are not corrected for humidity. The humidity is monitored and recorded at both facilities. The BIPM laboratory humidity is maintained at $50 \%$. The NIST laboratory average humidity is $30 \%$.

\section{Measurement Uncertainties}

The uncertainties associated with the primary standards are given in Table 6 . The NIST uncertainties were evaluated according to Ref. [6]. The uncertainties associated with the calibration of the transfer ionization chambers at the NIST and at the BIPM are listed in Table 7. The type A uncertainty of the ionization current includes a component for the reproducibility of calibrations at each laboratory. For the BIPM, this is $0.03 \%$, based on repeat calibrations of the NIST transfer chambers on different days. For the NIST, the value $0.12 \%$ is derived from the difference in the mean calibration coefficients before and after the measurements 
Volume 111, Number 5, September-October 2006

Journal of Research of the National Institute of Standards and Technology

Table 6. Relative standard uncertainties (in \%) associated with the standards

\begin{tabular}{lcccc}
\hline \hline Source of uncertainty & \multicolumn{2}{c}{ NIST } & \multicolumn{2}{c}{ BIPM } \\
& Type A & Type B & Type A & Type B \\
\hline Ionization current & 0.15 & 0.06 & 0.03 & 0.02 \\
Volume & 0.04 & 0.01 & 0.01 & 0.05 \\
Positioning & - & 0.01 & 0.01 & 0.01 \\
Correction factors (excl. $\left.k_{\mathrm{h}}\right)$ & 0.16 & 0.22 & 0.04 & 0.12 \\
Humidity $k_{\mathrm{h}}$ & - & 0.03 & - & 0.03 \\
Physical constants & - & 0.15 & - & 0.15 \\
\multicolumn{1}{c}{$\dot{K}_{\text {LAB }}$} & 0.23 & 0.27 & 0.05 & 0.20 \\
\hline
\end{tabular}

Table 7. Relative standard uncertainties (in \%) associated with the calibration of the transfer ionization chambers

\begin{tabular}{lcccc}
\hline \hline Source of uncertainty & \multicolumn{2}{c}{ NIST } & \multicolumn{2}{c}{ BIPM } \\
& Type A & Type B & Type A & Type B \\
\hline Air-kerma rate $\dot{K}_{\text {LAB }}$ & 0.23 & 0.27 & 0.05 & 0.20 \\
Ionization current & 0.12 & 0.06 & 0.03 & 0.02 \\
Positioning & - & 0.01 & 0.01 & 0.01 \\
$N_{K, \mathrm{LAB}}$ & 0.26 & 0.28 & 0.06 & 0.20 \\
\hline
\end{tabular}

at the BIPM. The uncertainty of the comparison results is given in Table 8 .

\section{Results and Discussion}

The results for the transfer ionization chamber calibrations at both laboratories are shown in Table 9. For each transfer chamber and each radiation quality, the ratio of the calibration coefficients $N_{K, \mathrm{NIST}} / N_{K, \mathrm{BIPM}}$ is evaluated, as shown in Table 10. Also given in the table is the standard uncertainty of the distribution for each set of three determinations. This does not represent the comparison uncertainty, but rather the consistency of the results determined with the different transfer chambers. There is no clear reason for the higher value of $0.29 \%$ at $250 \mathrm{kV}$, but the results at the other three qualities are consistent with the values determined for the

Table 8. Relative standard uncertainties (in \%) associated with the comparison results $R_{K}$

\begin{tabular}{|c|c|c|}
\hline Source of uncertainty & Type A & Type B \\
\hline & 0.27 & 0.25 \\
\hline
\end{tabular}

reproducibility of NIST calibrations $(0.12 \%)$ noted in the preceding section.

The comparison result $R_{K}$ for each radiation quality is the mean of the three ratios for that quality. These are given in Table 11 and show evidence of a trend with beam quality, decreasing by $0.8 \%$ between $100 \mathrm{kV}$ and $250 \mathrm{kV}$. This trend has been seen previously in certain comparisons at the BIPM and no reason has been found. One possibility under study is the dependence of the calibration coefficients on field size; the field sizes at the NIST $(60 \mathrm{~mm})$ and at the BIPM $(83 \mathrm{~mm})$ are quite different.

The comparison uncertainty given in Table 11 takes into account correlations in the BIPM and NIST values for the physical constants and the humidity correction. Correlations also exist in the values for $k_{\mathrm{e}}, k_{\mathrm{sc}}$, and $k_{\mathrm{fl}}$, since both laboratories use values derived from the same Monte Carlo calculations [7]. These are accounted for by taking half the uncertainty value for each component at each laboratory, in accordance with the analysis made for the degrees of equivalence appearing in the BIPM key comparison database. All other correction factors are assumed to be uncorrelated.

Comparing these results with those of the NIST/BIPM comparison of 1991, there is reasonable consistency within the uncertainties, except for a difference exceeding $0.5 \%$ at $100 \mathrm{kV}$. Although a number of 
Volume 111, Number 5, September-October 2006

Journal of Research of the National Institute of Standards and Technology

Table 9. Measured results for the calibration of the NIST transfer chambers at both laboratories

\begin{tabular}{|c|c|c|c|c|c|}
\hline \multirow[t]{2}{*}{$\begin{array}{l}\text { Reference } \\
\text { radiation }\end{array}$} & \multicolumn{2}{|c|}{$\begin{array}{l}\text { Half-value layer } \\
\text { (HVL) }\end{array}$} & \multicolumn{3}{|c|}{$\begin{array}{l}\text { NIST transfer chambers } \\
\text { Calibration coefficients } \\
\qquad\left(10^{6} \mathrm{~Gy} \mathrm{C}^{-1}\right)\end{array}$} \\
\hline & $\mathrm{mm} \mathrm{Al}$ & $\mathrm{mm} \mathrm{Cu}$ & NIST-T1 & NIST-T2 & NIST-T3 \\
\hline \multicolumn{6}{|l|}{ BIPM } \\
\hline $100 \mathrm{kV}$ & 4.027 & 0.148 & 8.382 & 8.469 & 7.965 \\
\hline $135 \mathrm{kV}$ & - & 0.494 & 8.477 & 8.539 & 8.080 \\
\hline $180 \mathrm{kV}$ & - & 0.990 & 8.608 & 8.658 & 8.182 \\
\hline $250 \mathrm{kV}$ & - & 2.500 & 8.768 & 8.813 & 8.290 \\
\hline \multicolumn{6}{|l|}{ NIST } \\
\hline $100 \mathrm{kV}$ & 3.943 & 0.149 & 8.388 & 8.465 & 7.983 \\
\hline $135 \mathrm{kV}$ & - & 0.496 & 8.440 & 8.492 & 8.056 \\
\hline $180 \mathrm{kV}$ & - & 1.003 & 8.576 & 8.619 & 8.164 \\
\hline $250 \mathrm{kV}$ & - & 2.502 & 8.704 & 8.727 & 8.257 \\
\hline
\end{tabular}

Table 10. Results of the comparison for each of the transfer chambers

\begin{tabular}{lccccc}
\hline \hline $\begin{array}{l}\text { Reference } \\
\text { radiation }\end{array}$ & NIST-T1 & NIST-T2 & NIST-T3 & $\begin{array}{c}\text { Mean for the transfer } \\
\text { chambers } \\
\text { mean } \\
N_{K, \text { NIST }} / N_{K, \mathrm{BIPM}} / N_{K, \mathrm{BIPM}}\end{array}$ & $\begin{array}{c}\text { Std unc of } \\
\text { distribution }\end{array}$ \\
\hline $100 \mathrm{kV}$ & 1.0008 & 0.9995 & 1.0022 & 1.0008 & $0.14 \%$ \\
$135 \mathrm{kV}$ & 0.9956 & 0.9944 & 0.9970 & 0.9957 & $0.13 \%$ \\
$180 \mathrm{kV}$ & 0.9962 & 0.9954 & 0.9978 & 0.9965 & $0.12 \%$ \\
$250 \mathrm{kV}$ & 0.9927 & 0.9903 & 0.9961 & 0.9930 & $0.29 \%$ \\
\hline
\end{tabular}

Table 11. Results $R_{K}$ of the comparison of the NIST and BIPM airkerma standards

\begin{tabular}{lccc}
\hline \hline $\begin{array}{l}\text { Reference } \\
\text { radiation }\end{array}$ & $\begin{array}{c}R_{K} \\
(2003)\end{array}$ & $\begin{array}{c}\text { Standard } \\
\text { uncertainty } \\
(\%)\end{array}$ & $\begin{array}{c}R_{K} \\
(1991)\end{array}$ \\
\hline $100 \mathrm{kV}$ & 1.0008 & 0.37 & 0.9952 \\
$135 \mathrm{kV}$ & 0.9957 & 0.37 & 0.9953 \\
$180 \mathrm{kV}$ & 0.9965 & 0.37 & 0.9942 \\
$250 \mathrm{kV}$ & 0.9930 & 0.37 & 0.9930 \\
\hline
\end{tabular}

corrections factors, for example $k_{\mathrm{e}}$ and $k_{\mathrm{sc}}$, have changed, and the factor $k_{\mathrm{fl}}$ has been introduced at both laboratories, the most notable change is in the air-attenuation correction at the NIST. For $100 \mathrm{kV}$, this factor was 1.0097 in $1991(\mathrm{HVL}=5 \mathrm{~mm} \mathrm{Al})$ and is now $1.0152(\mathrm{HVL}=3.943 \mathrm{~mm} \mathrm{Al})$, a change that accounts for most of the difference seen in the comparison results at $100 \mathrm{kV}$. For comparison, the BIPM value is $1.0102(\mathrm{HVL}=4.027 \mathrm{~mm} \mathrm{Al})$, which is much closer to the NIST value from 1991 than it is to the current NIST determination.

These results will be used as the basis of degrees of equivalence for the NIST medium-energy $\mathrm{x}$-ray standard in the BIPM key comparison database.

\section{References}

[1] BIPM, Qualités de rayonnements, Consultative Committee for Ionizing Radiation (CCEMRI) (Section I), 1972, 2, R15.

[2] M. Boutillon, Mesure de l'exposition au BIPM dans le domaine des rayons X de 100 à $250 \mathrm{kV}$, Rapport BIPM-78/3, 1978.

[3] M. Boutillon, Measuring Conditions Used for the Calibration of Ionization Chambers at the BIPM, Rapport BIPM-96/1, 1996.

[4] H. O. Wyckoff and F. H. Attix, Design of free-air ionization chambers, National Bureau Standards Handbook 64, (1957).

[5] P. J. Lamperti and M. OBrien, Calibration of X-Ray and GammaRay Measuring Instruments, NIST Special Publication 250-58, (2001).

[6] B. N. Taylor and C. E. Kuyatt, Guidelines for Evaluating and Expressing the Uncertainty of NIST Measurement Results, 1994 Edition, NIST Technical Note 1297, September 1994. 
Volume 111, Number 5, September-October 2006

Journal of Research of the National Institute of Standards and Technology

[7] D. T. Burns, Free-air chamber correction factors for electron loss, photon scatter, fluorescence and bremsstrahlung, CCRI(I)/01-36, 2001.

About the authors: Michelle O'Brien is a physicist in the Ionizing Radiation Division, Dosimetry and Interactions Group of the NIST Physics Laboratory. David Burns is a physicist in the Ionizing Radiation Section of the Bureau International des Poids et Mesures located in Sèvres, France. 